\title{
Photocatalytic Reductive Formation of $\alpha$-Tertiary Ethers from Ketals
}

Thomas Rossolini, ${ }^{\ddagger}$ Branislav Ferko, ${ }^{\ddagger \neq \neq}$ and Darren J. Dixon., ${ }^{\ddagger, *}$

‡ Department of Chemistry, University of Oxford, Chemistry Research Laboratory, 12 Mansfield Road, Oxford, OX1 3TA, UK

$¥$ Department of Organic Chemistry, Slovak University of Technology, Radlinského 9, 81237, Bratislava, Slovakia

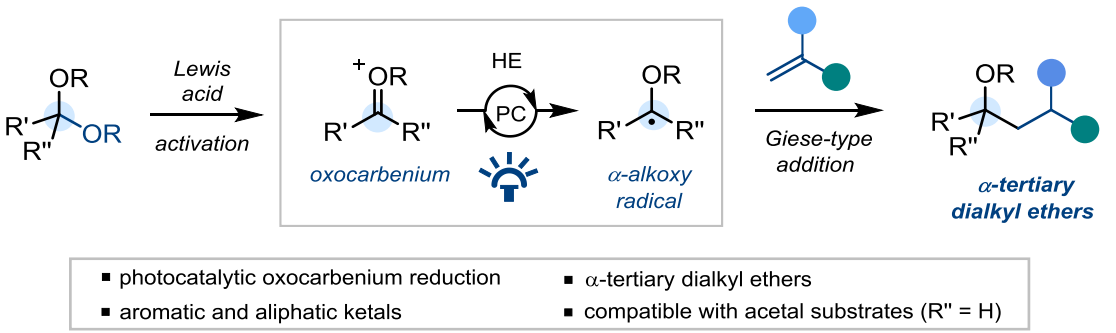

\begin{abstract}
A general photocatalytic reductive strategy for the construction of unsymmetrical $\alpha$-tertiary dialkyl ethers is reported. By merging Lewis acid-mediated ketal activation and visible-light photocatalytic reduction, in situ generated $\alpha$-alkoxy radicals can engage in addition reactions with a variety of olefinic partners. Good reaction efficiency is demonstrated with a range of ketals of aromatic and aliphatic ketones. Extension to acetal substrates is also described, demonstrating the overall synthetic utility of this methodology for complex ether synthesis.
\end{abstract}

Despite the widespread relevance of dialkyl ethers in pharmaceuticals and bioactive compounds, ${ }^{1}$ several challenges remain to access this structural unit. Approaches based upon the formation of the corresponding $\mathrm{C}\left(\mathrm{sp}^{3}\right)-\mathrm{O}$ bond - such as classical Williamson ether synthesis - are widely employed to access simple ethers, but are far from general for more complex systems. ${ }^{2}$ To this end, a number of key advances exploiting bespoke reagents ${ }^{3}$ and the intrinsic reactivity of various coupling partners ${ }^{4}$ have been made in recent times, leading to new valuable $\mathrm{C}-\mathrm{C}$ bond disconnections for ether construction.

Significant efforts in photoredox catalysis have offered mild and unique approaches for new reaction discovery via in situ generation of free-radical species which can partake in radical coupling or transition metal-catalyzed cross-coupling processes. ${ }^{5}$ In the context of ether synthesis, MacMillan and coworkers have reported an elegant synthetic procedure for the generation of benzhydryl ethers exploiting a dual photoredox/organocatalytic strategy via $\mathrm{C}-\mathrm{H}$ atom abstraction, utilizing cyanoarenes as coupling partners (Scheme 1a). ${ }^{6}$

Scheme 1. a) Reported methods for the synthesis of dialkyl ethers. b) Visible-light mediated direct strategy towards the formation of $\alpha$-tertiary dialkyl ethers from ketals.

a) C-C bond construction for the synthesis of dialkyl ethers (previous work)

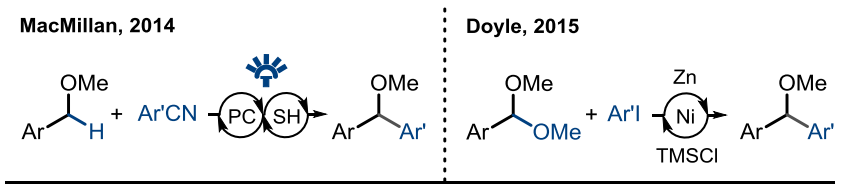

b) Photocatalytic reductive coupling of ketals/acetals (this work)

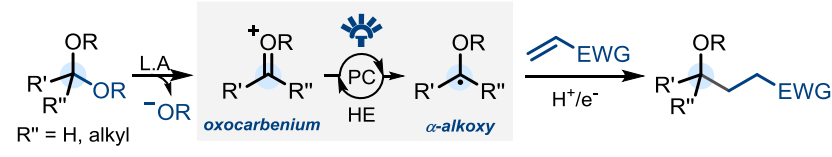

$\mathrm{PC}=$ photocatalyst, $\mathrm{HE}=$ Hantzsch ester.
More recently, the Doyle group has reported a powerful method to form similar products via a nickel-catalyzed reductive cross-coupling from acetals of aromatic aldehydes (Scheme 1a). ${ }^{7}$ Although the above chemistries have advanced carboncarbon bond-forming ether syntheses, ${ }^{8}$ new and general synthetic approaches for accessing $\alpha$-tertiary alkyl ethers remain desirable. To this end, and in an effort to expand the synthetic range of photocatalytic umpolung chemistry, ${ }^{9}$ we sought to explore alternative and common functional groups to engage in radical coupling reactions. ${ }^{10}$ We envisaged that ketal activation with a Lewis acid $^{7,11}$ followed by visible-light mediated single-electron transfer (SET) to the resulting oxocarbenium ion could deliver a putative nucleophilic $\alpha$ alkoxy radical intermediate, which could participate in radical addition chemistry (Scheme 1b). If successful, this mild generation and controlled coupling of the $\alpha$-alkoxy radical could provide direct access to $\alpha$-tertiary ethers in one step from readily available starting materials.

Preliminary evaluation of the proposed photocatalytic reductive coupling process was carried out with 4'fluoroacetophenone dimethyl ketal (1a), $\left[\operatorname{Ir}\left(\mathrm{dF}\left(\mathrm{CF}_{3}\right) \mathrm{ppy}\right)_{2}(\mathrm{dtbbpy})\right] \mathrm{PF}_{6}$, and dehydroalanine (DHA) derivative 2 as a coupling partner ${ }^{10 \mathrm{~d}, 12}$ (Scheme 2). Initial feasibility studies followed by reaction optimization elucidated that irradiation under blue LED light in DMA, with trimethylsilyl trifluoromethanesulfonate (TMSOTf) as Lewis acid and 4-phenyl Hantzsch ester $\left(\mathrm{HE}_{\mathrm{Ph}, \mathrm{Et}}\right)$ as stoichiometric reductant, provided the desired tertiary methyl ether $\mathbf{3}$ in an optimal $78 \%$ NMR yield (entry 1).

Observations made during reaction optimization revealed the importance of concentration and solvent selection on reaction outcome (see SI for more details). Significant enhancement was achieved employing higher substrate concentration, with DMA proving to be the most suitable medium (entry 1-3). Notably, and in contrast to previous reports, ${ }^{7,11}$ we recognized 
that trimethylsilyl chloride (TMSCl) was not an appropriate Lewis acid to promote ketal functionalization, as product $\mathbf{3}$ was afforded in negligible yield (entry 6). Additionally, Lewis acidic boron complexes, such as $\mathrm{BF}_{3}{ }^{\circ} \mathrm{OEt}_{2}$, were also found to be ineffective (entry 7). ${ }^{13}$

Scheme 2. Reaction Optimization.

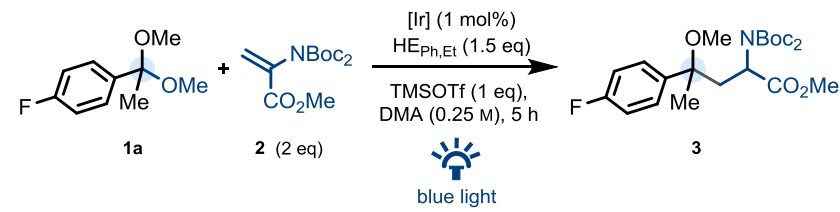

\begin{tabular}{c|c|c} 
Entry & Deviations from above & $\mathbf{3}(\%)^{\mathbf{a}}$ \\
\hline 1 & none & $78(66)^{\mathbf{b}}$ \\
2 & $0.2 \mathrm{M}$ in DMA & 65 \\
3 & $0.2 \mathrm{M}$ in DMSO & 56 \\
4 & $4-\mathrm{CzIPN}$ instead of [Ir] & 71 \\
5 & {$\left[\mathrm{Ir}(\mathrm{dFppy})_{2}(\mathrm{dtbbpy})\right]_{P F}$ instead of [Ir] } & 75 \\
$6^{\mathrm{c}}$ & $\mathrm{TMSCl}_{\mathrm{instead} \text { of TMSOTf }}$ & trace \\
$7^{\mathrm{c}}$ & $\mathrm{BF}_{3} \bullet \mathrm{OEt}_{2}$ instead of TMSOTf & trace \\
\hline
\end{tabular}

a: ${ }^{1} \mathrm{H}$ NMR yield based on 1,3,5-trimethoxybenzene as internal standard. b: Isolated yield. c: 0.2 $\mathrm{M}$ in DMSO. $[\mathrm{Ir}]=\left[\mathrm{Ir}\left(\mathrm{dF}\left(\mathrm{CF}_{3}\right) \mathrm{ppy}\right)_{2}(\mathrm{dtbbpy})\right] \mathrm{PF}_{6}, \mathrm{HE}_{\mathrm{Ph}, \mathrm{Et}}=$ diethyl 2,6-dimethyl-4-phenyl-1,4dihydropyridine-3,5-dicarboxylate, $\mathrm{DMA}=\mathrm{N}, \mathrm{N}$-dimethylacetamide.

With optimized conditions established, we sought to explore the scope of this photocatalytic carbon-carbon bond forming reductive coupling reaction with respect to the ketal moiety (Scheme 3A). ${ }^{14}$ Initially, substitution around the aromatic ring was investigated (3-8). Efficient reaction outcomes were observed with electron-releasing functional groups (5-6), supporting a nucleophilic character of the putative $\alpha$-alkoxy radical intermediate. ${ }^{12}$ Conversely, suppressed reactivity was observed with electron-withdrawing groups; where, for example a bromine atom in the para position afforded product $\mathbf{8}$ in $33 \%$.

Ketals bearing larger alkyl substituents underwent the reaction with good yields (9-10). The small drop in reactivity was consistent with the increasing steric hindrance compared to methyl-substituted 4 . $^{15}$ A chlorine-containing side chain was also tolerated and coupled product $\mathbf{1 1}$ was delivered in 36\% yield. Importantly, the substrate scope of this transformation was successfully expanded to diethyl and diisopropyl ketals in moderate to good yields (12-13), and this reactivity represents a significant improvement considering previously reported limitations on acyclic acetals. ${ }^{7}$ Moreover, ketals of aliphatic ketones were found to be competent substrates for radical generation, delivering the corresponding products in synthetically useful yields after conjugate addition (14-16), with dimethoxy cyclopentane affording compound $\mathbf{1 7}$ in $53 \%$ yield. In order to demonstrate the generality of this transformation, we looked to expand the scope to include acetal substrates. Pleasingly, good reaction efficiency was achieved with both acyclic and cyclic acetals (18-19), illustrating complementarity with previous works. ${ }^{7,11 b}$ Finally, the role of the alkoxide functionality was examined with mixed ketal 1s (Scheme 3B). Interestingly, phenyl ether 14' was not formed under the reaction conditions, however methyl ether 14 was isolated in 38\% yield (comparable to dimethoxy propane ketal as starting material, Scheme $3 \mathrm{~A}, 14)$. This observation could be attributed to the better leaving group ability of the phenoxy group compared to the methoxy group.
We next examined the scope of this reaction with respect to the acceptor component, using ketal 1a as model substrate (Scheme 4A). It should be noted that in order to further improve reactivity with acrylate-type acceptors, we investigated alternative reagents to favor radical termination after Giesetype addition. In contrast to a radical reduction/protonation mechanism for DHA, a different termination via hydrogen atom transfer (HAT) has been proposed previously for acrylate substrates. ${ }^{16}$ Catalytic methods involving thiols were not found to be viable for this purpose. ${ }^{16,17}$ However, $\mathrm{H}$-atom abstraction from stoichiometric 1,4-cyclohexadiene (CHD) proved to be beneficial in improving reaction yields (see SI for details). ${ }^{18}$ Using these modified conditions, photocatalytic reductive coupling was achieved between 1a, phenyl methacrylate, and methyl methacrylate to give the corresponding tertiary methyl ethers in $74 \%$ and $62 \%$ yield, respectively (20-21). Variations to the $\alpha$-position of the acrylate component were found to play a pivotal role in reactivity. Pleasingly, fluorine incorporation was tolerated; mono-fluorinated $\mathbf{2 2}$ and trifluoromethylated $\mathbf{2 3}$ were both afforded in synthetically useful yields. No gemdifluoroalkene adducts were formed under these reaction conditions. ${ }^{11 b, 19}$ Unsubstituted phenyl acrylate resulted in suppressed efficiency even when a large excess of coupling partner was used (24). Furthermore, coupling with methacryloyloxazolidinone furnished $\mathbf{2 5}$ with moderate yield. These results indicate a key stabilizing captodative effect arising from the $\alpha$-substituent, a conclusion further supported by successful coupling with electron neutral alkenes. In fact, significant efficiency was observed with diphenylethylene as the acceptor olefin (26).

Under these reaction conditions reductive allylation of ketal 1a with a sulfone derivative afforded compound $\mathbf{2 7}$ in $30 \%$, despite the different reaction mechanism involved with these coupling partners. $^{9 \mathrm{~d}, 9 \mathrm{e}}$

Finally, 1a was subjected to reaction conditions without an acceptor partner, which afforded dimerized aryl methyl ether 28 in $52 \%$ yield as a 1:1 mixture of diastereomers. Rueping and coworkers have reported the reductive dimerization of aldehydes and ketones via ketyl radical generation under visible light irradiation. ${ }^{20}$ Accordingly, this result supports a radical pinacol-type dimerization and provides direct access to ether pinacol adducts from easily accessible starting materials.

Control experiments established the essential role of the Lewis acid; no conversion of starting material was observed without TMSOTf (see SI for details). Moreover, none of the desired reactivity was achieved in the absence of light, photocatalyst, or Hantzsch ester. On the basis of these results, we propose the following reaction mechanism (Scheme 4B). ${ }^{13}$ Initially, ketal 1 is activated by TMSOTf to form the oxocarbenium ion intermediate $\mathbf{I}$ as disclosed in previous reports. ${ }^{7,11}$ A highly reducing Ir $^{\text {II }}$ species $\left(E_{1 / 2 \text { red }}=-1.37 \mathrm{~V}\right.$ vs. SCE in MeCN), ${ }^{5 b}$ generated by electron transfer from the Hantzsch ester following photoexcitation of $\mathrm{Ir}^{\mathrm{III}}$, is capable of reducing $\mathbf{I}$ to generate the $\alpha$ alkoxy radical II. ${ }^{21}$ The key nucleophilic intermediate (I) can engage in radical addition with an appropriate acceptor olefin moiety. After this Giese-type addition, the resulting free radical intermediate (III) undergoes a reduction/protonation sequence mediated by the Hantzsch ester $\left(\mathrm{HE}^{\bullet}\right)$, or HAT termination favored by the presence of CHD, depending on the nature of the coupling partner. ${ }^{16}$ 
Scheme 3. (A) Scope with respect to the ketal substrate. (B) Performance evaluation of mixed ketal substrate 1s.

(A)
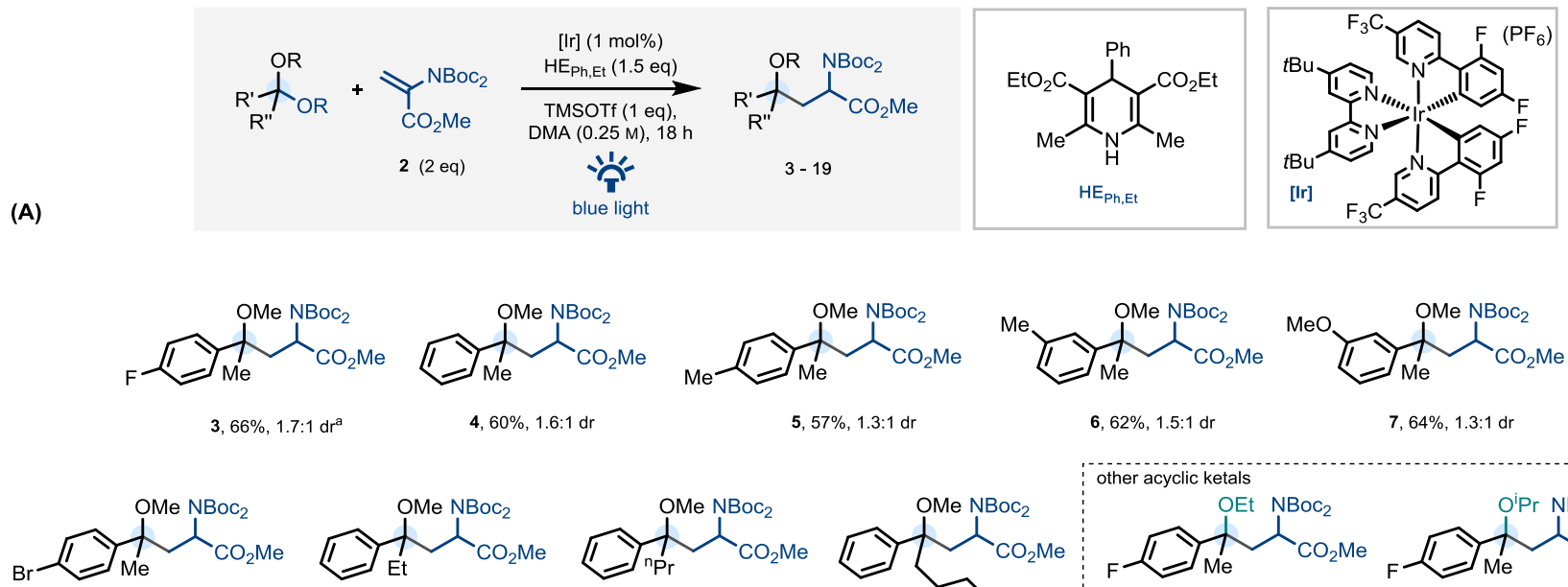

$8,33 \%, 1.2: 1 \mathrm{dr}$

$9,54 \%, 1.2: 1 \mathrm{dr}$

$10,50 \%, 1.5: 1 \mathrm{dr}$

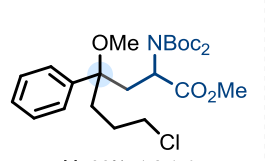

other acyclic ketals

$7,64 \%, 1.3: 1 \mathrm{dr}$
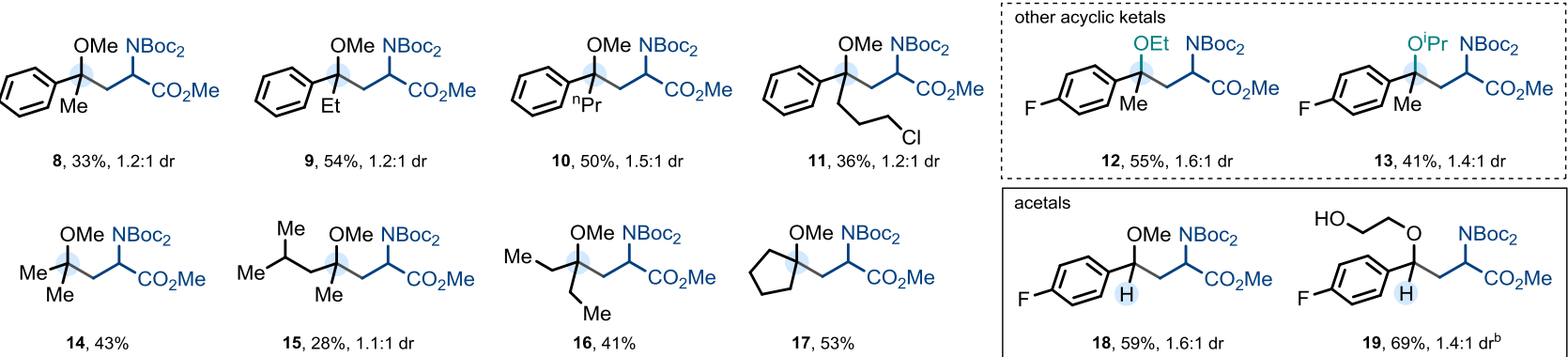

(B)
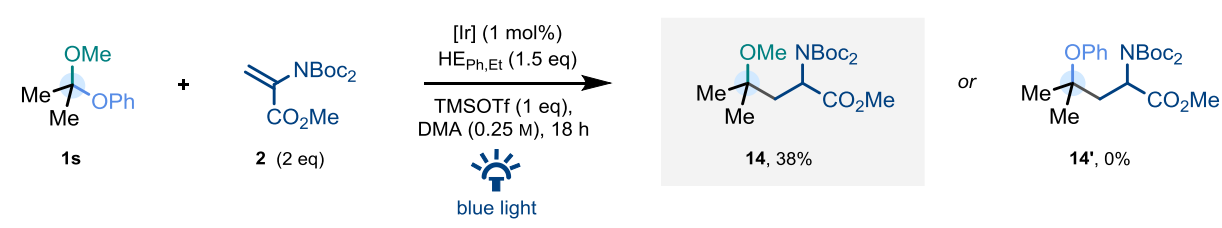

Combined isolated yields on $0.2 \mathrm{mmol} \mathrm{scale} .{ }^{\mathrm{a}}$ Reaction time $5 \mathrm{~h} .{ }^{\mathrm{b}}$ Reaction time $48 \mathrm{~h}$.

In summary, the photocatalytic reductive coupling of easily accessible ketals and acceptor olefins has been developed. This methodology provides a new approach towards generating challenging $\alpha$-tertiary dialkyl ethers in a single step, and is also amenable to acetal components. Extension to pinacol coupling adducts via an $\alpha$-alkoxy radical dimerization process has been demonstrated thus providing insight into the mechanism. We believe that this methodology provides a complementary new approach to access complex $\alpha$-tertiary ether architectures, and that the photocatalytic SET reduction of oxocarbenium ions will provide new opportunities for future reaction development. 
Scheme 4. (A) Scope with respect to the alkene coupling partner. (B) Plausible reaction mechanism.

(A)<smiles>COC(C)(C)c1ccc(F)cc1</smiles>

$1 \mathrm{a}$

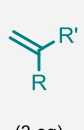

(3 eq)

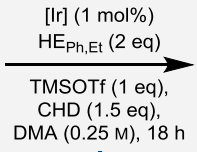

"'ै"

blue light

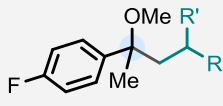

$20-27$<smiles>CCOC(=O)C1=C(C(=O)OCC)C(c2ccccc2)C([N+](=O)[O-])=C(C(=O)OCC)C1=O</smiles>

$H E_{\mathrm{Ph}, \mathrm{Et}}$
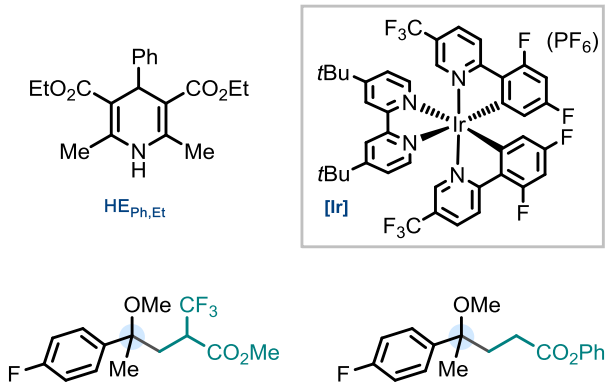

$23,43 \%, 1.3: 1 \mathrm{dr}$

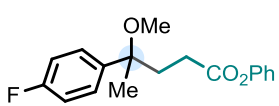

$24,22 \%\left(25 \%{ }^{\mathrm{a}}\right)$ 20, $74 \%, 1.2: 1 \mathrm{dr}$
$(1 \mathrm{mmol}: 71 \%, 1.2: 1)$

$21,62 \%, 1.1: 1 \mathrm{dr}$

22, $45 \%, 1.3: 1 \mathrm{dr}$<smiles>C=C(CC(C)(OC)c1ccc(F)cc1)C(=O)OCC</smiles>

$27,30 \%{ }^{\mathrm{c}}$ from allyl sulfone
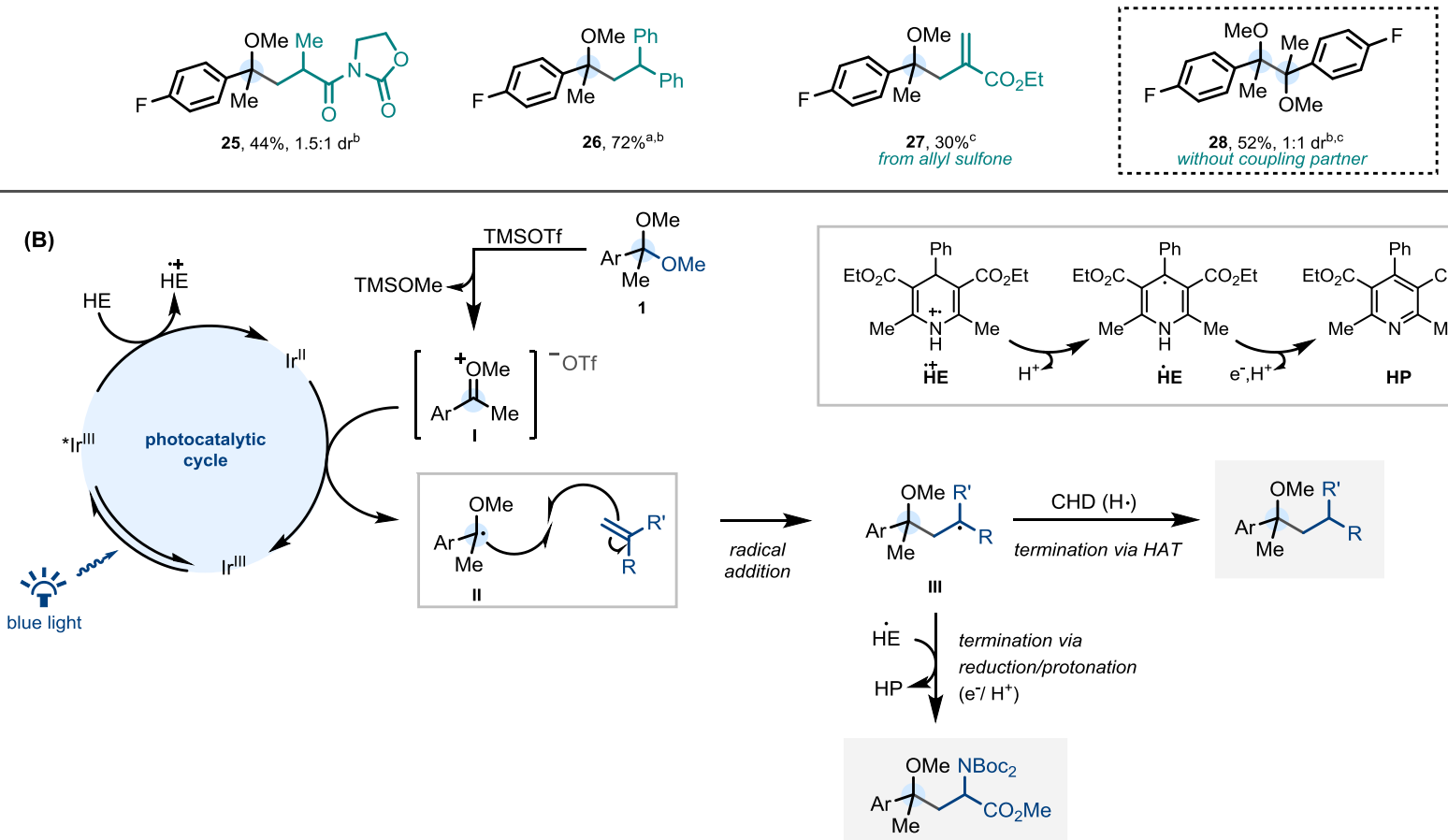

$\left(\mathrm{R}=\mathrm{CO}_{2} \mathrm{Me}, \mathrm{R}^{\prime}=\mathrm{NBoc}_{2}\right)$

Combined isolated yields on $0.2 \mathrm{mmol}$ scale. $\mathrm{CHD}=1,4$-cyclohexadiene. ${ }^{\mathrm{a}} 10$ eq of coupling partner. ${ }^{\mathrm{b}}$ Reaction performed on 0.1 mmol scale. ${ }^{\mathrm{c}}$ Reaction performed without $\mathrm{CHD}$.

\section{ASSOCIATED CONTENT}

The Supporting Information is available free of charge on the ACS Publications website. Synthetic procedures and full characterization data of compounds (PDF).

\section{AUTHOR INFORMATION}

\section{Corresponding Author}

* Darren J. Dixon: darren.dixon@ chem.ox.ac.uk

\section{ACKNOWLEDGMENT}

DJD and TR wish to thank Astex Pharmaceuticals, Ltd. for generous financial support, Dr. Steven Howard (Astex Pharmaceuticals, Ltd.), and Dr. Rachel Grainger (Astex Pharmaceuticals, Ltd.) for helpful discussion. BF is thankful to the Slovak Academic Information Agency (SAIA) for his research mobility grant. We thank Tatiana Rogova (University of Oxford) for providing computational data and Prof. Fernanda Duarte (University of Oxford) for advice and supervision.

\section{REFERENCES}

(1) (a) De Martino, G.; La Regina, G.; Di Pasquali, A.; Ragno, R.; Bergamini, A.; Cipriani, C.; Sinistro, A.; Maga, G.; Crespan, E.; Artico, M.; Silvestri, R. Novel 1-[2-(Diarylmethoxy)ethyl]-2-methyl-5nitroimidazoles as HIV-1 Non-Nucleoside Reverse Transcriptase Inhibitors. A Structure-Activity Relationship Investigation. J. Med. Chem. 2005, 48, 4378-4388; (b) Schmidt, F.; Stemmler, R. T.; Rudolph, J.; Bolm, C. Catalytic asymmetric approaches towards enantiomerically enriched diarylmethanols and diarylamines. Chem. Soc. Rev. 2006, 35, 454-470; (c) Eildal, J. N. N.; Andersen, J.; Kristensen, A. S.; Jørgensen, A. M.; Bang-Andersen, B.; Jørgensen, M.; Strømgaard, K. From the Selective Serotonin Transporter Inhibitor Citalopram to the Selective Norepinephrine Transporter Inhibitor Talopram: Synthesis and Structure-Activity Relationship Studies. J. Med. Chem. 2008, 51, 3045-3048; (d) Roughley, S. D.; Jordan, A. M. The Medicinal Chemist's Toolbox: An Analysis of Reactions Used in the Pursuit of Drug Candidates. J. Med. Chem. 2011, 54, 3451-3479; (e) 
Ameen, D.; Snape, T. J. Chiral 1,1-diaryl compounds as important pharmacophores. Med. Chem. Commun. 2013, 4, 893-907.

(2) (a) Williamson, A. W. On Etherification. Q. J. Chem. Soc., 1852, 4, 229-239; (b) Barluenga, J.; Tomás-Gamasa, M.; Aznar, F.; Valdés, C. Straightforward Synthesis of Ethers: Metal-Free Reductive Coupling of Tosylhydrazones with Alcohols or Phenols. Angew. Chem. Int. Ed. 2010, 49, 4993-4996; (c) Blackmond and Baran have recently reported a new $\mathrm{C}-\mathrm{O}$ bond forming approach based on electrogenerated carbocations: Xiang, J.; Shang, M.; Kawamata, Y.; Lundberg, H.; Reisberg, S. H.; Chen, M.; Mykhailiuk, P.; Beutner, G.; Collins, M. R.; Davies, A.; Del Bel, M.; Gallego, G. M.; Spangler, J. E.; Starr, J.; Yang, S.; Blackmond, D. G.; Baran, P. S. Hindered Dialkyl Ether Synthesis via Electrongenerated Carbocations. ChemRxiv 2019, DOI: 10.26434/chemrxiv.8144294.v1

(3) (a) Molander, G. A.; Wisniewski, S. R. Stereospecific CrossCoupling of Secondary Organotrifluoroborates: Potassium 1-(Benzyloxy)alkyltrifluoroborates. J. Am. Chem. Soc. 2012, 134 16856-16868; (b) Vo, C.-V. T.; Mitchell, T. A.; Bode, J. W. Expanded Substrate Scope and Improved Reactivity of Ether-Forming CrossCoupling Reactions of Organotrifluoroborates and Acetals. J. Am. Chem. Soc. 2011, 133, 14082-14089.

(4) Moquist, P. N.; Kodama, T.; Schaus, S. E. Enantioselective Addition of Boronates to Chromene Acetals Catalyzed by a Chiral Brønsted Acid/Lewis Acid System. Angew. Chem. Int. Ed. 2010, 49, 7096-7100.

(5) For selected reviews see: (a) Narayanam, J. M. R.; Stephenson, C. R. J. Visible light photoredox catalysis: applications in organic synthesis. Chem. Soc. Rev. 2011, 40, 102-113; (b) Prier, C. K.; Rankic, D. A.; MacMillan, D. W. C. Visible Light Photoredox Catalysis with Transition Metal Complexes: Applications in Organic Synthesis. Chem. Rev. 2013, 113, 5322-5363; (c) Skubi, K. L.; Blum, T. R.; Yoon, T. P. Dual Catalysis Strategies in Photochemical Synthesis. Chem. Rev. 2016, 116, 10035-10074; (d) Twilton, J.; Lee, C.; Zhang, P.; Shaw, M. H.; Evans, R. W.; MacMillan, D. W. C. The merger of transition metal and photocatalysis. Enhancing the potential of enantioselective organocatalysis with light. Nat. Rev. Chem. 2017, 1, 0052; (e) Silvi, M.; Melchiorre, P. Nature 2018, 554, 41-49.

(6) (a) Qvortrup, K.; Rankic, D. A.; MacMillan, D. W. C. A General Strategy for Organocatalytic Activation of $\mathrm{C}-\mathrm{H}$ Bonds via Photoredox Catalysis: Direct Arylation of Benzylic Ethers. J. Am. Chem. Soc. 2014, 136, 626-629; (b) For $\beta$-amino ether synthesis see: Hager, D.; MacMillan, D. W. C. Activation of $\mathrm{C}-\mathrm{H}$ Bonds via the Merger of Photoredox and Organocatalysis: A Coupling of Benzylic Ethers with Schiff Bases. J. Am. Chem. Soc. 2014, 136, 16986-16989; (c) For another designed example of ether synthesis via $\mathrm{C}-\mathrm{H}$ atom abstraction see: Zhang, J.; Li, Y.; Zhang, F.; Hu, C.; Chen. Y. Generation of Alkoxyl Radicals by Photoredox Catalysis Enables Selective C $\left(\mathrm{sp}^{3}\right)-\mathrm{H}$ Functionalization under Mild Reaction Conditions. Angew. Chem. Int. Ed. 2016, 55, 1872-1875.

(7) Arendt. K. M.; Doyle, A. G. Dialkyl Ether Formation by Nickel-Catalyzed Cross-Coupling of Acetals and Aryl Iodides. Angew. Chem. Int. Ed. 2015, 54, 9876-9880.

(8) (a) MacMillan and co-workers have reported access to allylic ethers via decarboxylative coupling of $\alpha$-oxy carboxylic acids with vinyl halides: Noble, A.; McCarver, S. J.; MacMillan, D. W. C. Merging Photoredox and Nickel Catalysis: Decarboxylative CrossCoupling of Carboxylic Acids with Vinyl Halides. J. Am. Chem. Soc. 2015, 137, 624-627; for other methods involving $\alpha$-oxy radical generation see: (b) Ramirez, N. P.; Gonzalez-Gomez, J. C. Decarboxylative Giese-Type Reaction of Carboxylic Acids Promoted by Visible Light: A sustainable and Photoredox-Neutral Protocol. Eur. J. Org. Chem. 2017, 2154-2163; (c) Barton, D. H. R.; Gateau-Olesker, A.; Géro, S D.; Lacher, B.; Tachdjian, C.; Zard, S. Z. Radical Decarboxylative Alkylation of Tartaric Acid. Tetrahderon 1993, 49, 4589-4602; (d) Matsuda, F.; Kawatsura, M.; Hosaka, K.; Shirahama, H. HydroxylDirected Intramolecular Ketone-Olefin Couplings Promoted by $\mathrm{SmI}_{2}$. Chem. Eur. J. 1999, 5, 3252-3259.

(9) For imine umpolung chemistry see: (a) Rono, L. J.; Yayla, H. G.; Wang, D. Y.; Armstrong, M. F.; Knowles, R. R. Enantioselective Photoredox Catalysis Enabled by Proton-Coupled Electron Transfer: Development of an Asymmetric Aza-Pinacol Cyclization. J. Am.
Chem. Soc. 2013, 135, 17735-17738; (b) Jeffrey, J. L.; Petronijević, F. R.; MacMillan, D. W. C. Selective Radical-Radical CrossCouplings: Design of a Formal $\beta$-Mannich Reaction. J. Am. Chem. Soc. 2015, 137, 8404-8407; (c) Uraguchi, D.; Kinoshita, N.; Kizu, T.; Ooi, T. Synergistic Catalysis of Ionic Brønsted Acid and Photosensitizer for a Redox Neutral Asymmetric $\alpha$-Coupling of N-Arylaminomethanes with Aldimines. J. Am. Chem. Soc. 2015, 137, 13768-13771; (d) Qi, L.; Chen, Y. Polarity-Reversed Allylations of Aldehydes, Ketones, and Imines Enabled by Hantzsch Ester in Photoredox Catalysis. Angew. Chem. Int. Ed. 2016, 55, 13312-13315; (e) Fuentes de Arriba, A. L.; Urbitsch, F.; Dixon, D. J. Umpolung synthesis of branched a-functionalized amines from imines via photocatalytic threecomponent reductive coupling reactions. Chem. Commun. 2016, 52, 14434-14437; (f) Fava, E; Millet, A.; Nakajima, M.; Loescher, S.; Rueping, M. Reductive Umpolung of Carbonyl Derivatives with Visible-Light Photoredox Catalysis: Direct Access to Vicinal Diamines and Amino Alcohols via a-Amino Radicals and Ketyl Radicals. Angew. Chem. Int. Ed. 2016, 55, 6776-6779; (g) Lee, K. N; Lei, Z.; Ngai, M.-Y. $\beta$-Selective Reductive Coupling of Alkenylpyridines with Aldehydes and Imines via Synergistic Lewis Acid/Photoredox Catalysis. J. Am. Chem. Soc. 2017, 139, 5003-5006; (h) Leitch, J. A.; Fuentes de Arriba, A. L.; Tan, J.; Hoff, O.; Martinez, C. M.; Dixon, D. J. Photocatalytic reverse polarity Povarov reaction. Chem. Sci. 2018, 9, 6653-6658; (i) Trowbridge, A.; Reich, D.; Gaunt, M. J. Multicomponent synthesis of tertiary alkylamines by photocatalytic olefin-hydroaminoalkylation. Nature 2018, 561, 522-527; (j) Dhananjayan, V.; Fuentes de Arriba, A. L.; Leitch, J. A.; de Gombert, A; Dixon, D. J. Primary a-tertiary amine synthesis via a-C-H functionalization. Chem. Sci. 2019, 10, 3401-3407; For aldehyde and ketone umpolung chemistry see: (k) Ischay, M. A.; Anzovino, M. E.; $\mathrm{Du}, \mathrm{J}$; Yoon, T. P. Efficient Visible Light Photocatalysis of [2+2] Enone Cycloadditions. J. Am. Chem. Soc. 2008, 130, 12886-12887; (1) Tarantino, K. T.; Liu, P.; Knowles, R. R. Catalytic Ketyl-Olefin Cyclizations Enabled by Proton-Coupled Electron Transfer. J. Am. Chem. Soc. 2013, 135, 10022-10025; (m) Wang, C.; Qin, J.; Shen, X.; Riedel, R.; Harms, K.; Meggers, E. Asymmetric Radical-Radical Cross-Coupling through Visible-LightActivated Iridium Catalysis. Angew. Chem. Int. Ed. 2016, 56, 685-688; (n) Chen, M.; Zhao, X.; Yang, C.; Xia, W. Visible-Light-Triggered Directly Reductive Arylation of Carbonyl/ Iminyl Derivatives through Photocatalytic PCET. Org. Lett. 2017, 19, 3807-3810; (o) Berger, A. L.; Donabauer, K; König, B. Photocatalytic Barbier reaction - visible-light induced allylation and benzylation of aldehydes and ketones. Chem. Sci. 2018, 9, 7230-7235; (p) Wang, L.; Lear, J. M.; Rafferty, S. M.; Fosu, S. C.; Nagib, D. A. Ketyl radical reactivity via atom transfer catalysis. Science 2018, 362, 225-229.

(10) For selected publications see: (a) Nawrat, C. C.; Jamison, C. R; Slutskyy, Y.; MacMillan, D. W. C.; Overman, L. E. Oxalates as Activating Groups for Alcohols in Visible Light Photoredox Catalysis: Formation of Quaternary Centers by RedoxNeutral Fragment Coupling. J. Am. Chem. Soc. 2015, 137, 11270-11273; (b) Choi, G. J.; Zhu, Q.; Miller, D. C.; Gu, C. J.; Knowles, R. R. Catalytic alkylation of remote $\mathrm{C}-\mathrm{H}$ bonds enabled by proton-coupled electron transfer. Nature 2016, 539, 268-271; (c) Patel, N. R.; Kelly, C. B.; Siegenfeld, A. P.; Molander, G. A. Mild, Redox-Neutral Alkylation of Imines Enabled by an Organic Photocatalyst. ACS Catal. 2017, 7, 1766-1770; (d) Aycock, R. A.; Vogt, D. B.; Jui, N. T. A practical and scalable system for heteroaryl amino acid synthesis. Chem. Sci. 2017, 8, 79988003; (e) Shu, C.; Mega, R. S.; Andreassen, B. J.; Noble, A.; Aggarwal, V. K. Synthesis of Functionalized Cyclopropanes from Carboxylic Acids by a Radical Addition-Polar Cyclization Cascade. Angew. Chem. Int. Ed. 2018, 57, 15430-15434.

(11) For acetals activation by Lewis acids see: (a) Hatano, B.; Nagahashi, K; Habaue, S. Reductive Coupling of Aromatic Dialkyl Acetals Using the Combination of Zinc and Chlorotrimethylsilane in the Presence of Potassium Carbonate. Chem. Lett. 2007, 36, 1418-1419; (b) Lin, Z.; Lan, Y.; Wang, C. Synthesis of gem-Difluoroalkenes via Nickel-Catalyzed Reductive $\mathrm{C}-\mathrm{F}$ and $\mathrm{C}-\mathrm{O}$ Bond Cleavage. ACS Catal. 2019, 9, 775-780. 
(12) Rossolini, T.; Leitch, J. A.; Grainger, R.; Dixon, D. J. Photocatalytic Three-Component Umpolung Synthesis of 1,3- Diamines. Org. Lett. 2018, 20, 6794-6798.

(13) See Supporting Information (SI) for further details.

(14) Model substrate showed full conversion after $5 \mathrm{~h}$. However, to ensure consistency for all starting materials, reactions were run for $18 \mathrm{~h}$.

(15) Ketals of $\alpha$-branched ketones as well as heterocyclecontaining derivatives were not reactive under these conditions.

(16) Seath, C. P.; Vogt, D. B.; Xu, Z.; Boyington, A. J.; Jui, N. T. Radical Hydroarylation of Functionalized Olefins and Mechanistic Investigation of Photocatalytic Pyridyl Radical Reactions. J. Am. Chem. Soc. 2018, 140, 15525-15534.

(17) (a) Musacchio, A. J.; Lainhart, B. C.; Zhang, X.; Naguib, S. G.; Sherwood, T. C.; Knowles, R. R. Catalytic intermolecular hydroaminations of unactivated olefins with secondary alkyl amines. Science 2017, 355, 727-730; (b) Zhu, Q.; Graff, D. E.; Knowles, and R. R. Intermolecular Anti-Markovnikov Hydroamination of Unactivated Alkenes with Sulfonamides Enabled by Proton-Coupled Electron Transfer. J. Am. Chem. Soc. 2018, 140, 741-747; (c) Nguyen, S. T; Zhu, Q.; Knowles, R. R. PCET-Enabled Olefin Hydroamidation Reactions with N-Alkyl Amides. ACS. Cat. 2019, 9, 4502-4507.

(18) (a) Davies, J; Svejstrup, T. D.; Reina, D. F.; Sheikh, N. S.; Leonori, D. Visible-Light-Mediated Synthesis of Amidyl Radicals: Transition-Metal-Free Hydroamination and $N$-Arylation Reactions. $J$. Am. Chem. Soc. 2016, 138, 8092-8095; (b) Schweitzer-Chaput, B.; Horwitz, M. A; de Pedro Beato, E.; Melchiorre, P. Photochemical generation of radicals from alkyl electrophiles using a nucleophilic organic catalyst. Nat. Chem. 2019, 11, 129-135.

(19) Lang, S. B.; Wiles, R. J.; Kelly, C. B.; Molander, G. A. Photoredox Generation of Carbon-Centered Radicals Enables the Construction of 1,1-Difluoroalkene Carbonyl Mimics. Angew. Chem. Int. Ed. 2017, 56, 15073-15077.

(20) Nakajima, M.; Fava, E.; Loescher, S.; Jiang, Z.; Rueping, M. Photoredox-Catalyzed Reductive Coupling of Aldehydes, Ketones, and Imines with Visible Light. Angew. Chem. Int. Ed. 2015, 54, 88288832.

(21) The TfOH would be neutralized by the Hantzsch pyridine (HP) generated in the reaction. 